\title{
Keiner spricht über Pregabalin, warum nicht?
}

Fragestellung: Zu Pregabalin (PRG) gibt es bislang nur wenig oder nicht ausreichend in der öffentlichen Wahrnehmung angekommene Informationen über ein mögliches Missbildungsrisiko vor allem im ersten Trimenon.

Hintergrund: PRG ist ein weitverbreitetes Medikament, das nur in geringem Umfang - in Deutschland eigentlich sogar fast gar nicht mehr in der Behandlung von Epilepsien eingesetzt wird. Viel häufiger, geradezu regelmäßig ist hingegen der Griff zu PRG bei Schmerzen im Allgemeinen und im Speziellen bei sämtlichen neuropathischen Schmerzsyndromen. Außerdem hat es seine Indikationen in der Psychiatrie. In einigen Ländern ist es der Klassiker in der Behandlung generalisierter Angsterkrankungen geworden. Nationale Gesundheitsdaten unterschiedlichster Länder belegen, dass dabei vor allem Frauen PRG verordnet bekommen, in Schweden sind es zum Beispiel 63\%. Auf der anderen Seite wissen wir aus den Tierstudien, dass PRG ein toxisches Agens in der Entwicklung der Föten mit hoher Wahrscheinlichkeit für Skelettmissbildungen, Wachstumsverzögerungen, Neuralrohrdefekten und Spontanaborten ist. Das sind keine beruhigenden Daten.

Patienten und Methodik: Die vorliegende multizentrische prospektive Studie aus sieben Ländern verglich Frauen, die während der Schwangerschaft PRG einnahmen, mit denen

Winterfeld U, Merlob P, Baud D, Rousson $V$ et al. Pregnancy outcome following maternal exposure to pregabalin may call for concern. Neurology 2016; 86: $2251-7$ wohnheiten, unter anderem Tabak- oder Alkoholkonsum, miterfasst. Die Daten der unter PRG-Exposition geborenen Kinder wurden im Hinblick auf mögliche Teratogenität anhand entsprechender Register (Teratology Information Services, TIS) zwischen 2004 bis 2013 gesammelt und ausgewertet. Chromosomale Syndrome und genetische Defekte wurden von der Bewertung ausgeschlossen.

Ergebnisse: Insgesamt wurden im genannten Zeitraum 164 Schwangerschaften, die PRG-exponiert waren, im Vergleich zu 656 Kontrollen erfasst und ausgewertet. Die Hauptindikation für die regelmäßige PRG-Einnahme war Schmerz inklusive Migräne (122/164), gefolgt von psychiatrischen Störungen (Angst, bipolare Erkrankungen, Depression, Psychosen etc.) und in seltenen Fällen Schlafstörungen oder Epilepsie (je 5/164). Die mittlere Tagesdosis betrug $150 \mathrm{mg}$. Die Rate an Lebendgeborenen war in der PRG-Gruppe auffallend geringer (71,9\% vs. 85,2\%), dies letztlich auch durch vorzeitig beendete Schwangerschaften, aus medizinischer Indikation immerhin in 5,5\% vs. 1,8\% der Fälle. Die großen Geburtsfehler lagen mit 6,4\% deutlich über den 1,9\% der Kontrollgruppe ohne PRG. Betrachtet man alle Geburtsfehler, auch die der Kinder aus vorzeitig beendeten Schwangerschaften und der Totgeburten, lag das Missbildungsrisiko sogar bei 9,6\% vs. 2,8\%. Das Geburtsgewicht der Kinder war im Wesentlichen in beiden Gruppen gleich. Allerdings zeigten einige Kinder, deren Mütter bis zur Entbindung PRG eingenommen hatten, nach der Geburt Entzugssymptome oder Intoxikationszeichen.

Schlussfolgerungen: PRG ist mit einem relevant erhöhten Missbildungsrisiko vor allem für große Geburtsfehler (6,4\%) nach Exposition im ersten Trimenon behaftet.

\section{- Kommentar Vivien Homberg, Bad Berka}

\section{Pregabalin tut immer so harmlos, ist es aber nicht}

Hätte man die Tiermodelldaten aus den Zulassungsstudien beachtet, wären die hier erhobenen Daten keine echte Überraschung geworden, denn leider sind die damaligen Daten auf den Menschen übertragbar. Wie so oft hat aber keiner richtig hingeschaut oder es wahrhaben wollen. Festzuhalten ist, dass PRG überhaupt kein sinnvoller Partner in der Behandlung junger Frauen in gebärfähigem Alter ist. Warum tauchen die Ergebnisse erst jetzt auf? Dies ist wohl der Tatsache geschuldet, dass PRG nicht über die klassischen, extrem gut gepflegten Schwangerschaftsregister, in die seit vielen Jahren zum Beispiel Epilepsiepatientinnen aufgenommen werden, erfasst wird, da es bei dieser ursprünglich beworbenen Zulassung kaum mehr zum Einsatz kommt.

Relevante Daten, auch wenn die Autoren selber zurecht die insgesamt kleine Patientenanzahl monieren. Relevant deshalb, da nun eine ganz andere Patientengruppe bei einer Schwan- gerschaft in den Fokus eines strengen Monitorings rückt: die junge Schmerzpatientin oder die psychiatrisch Erkrankte, inklusive derer die wegen einer vielleicht sogar banalen Angststörung Medikamente erhalten, bevor andere therapeutische Optionen ausgereizt sind.

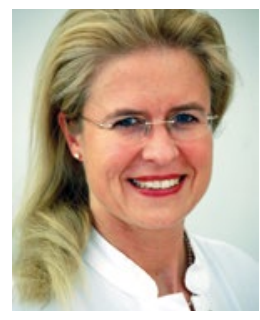

Dr. med. Vivien Homberg, Bad Berka

Chefärztin der Klinik für Neurologie, Zentralklinikum Bad Berka E-Mail:Vivien.Homberg@zentralklinik.de 